\title{
2. SYNOPSIS
}

\begin{tabular}{|c|c|c|}
\hline \multicolumn{2}{|c|}{ Name of Sponsor: I.R.I.S., 50 rue Carnot - 92284 Suresnes Cedex - France } & \multirow{2}{*}{$\begin{array}{l}\text { (For National } \\
\text { Authority Use only) }\end{array}$} \\
\hline $\begin{array}{l}\text { Test drug } \\
\text { Name of Finished Product: }\end{array}$ & & \\
\hline \multicolumn{2}{|c|}{ Name of Active Ingredients: Atorvastatin/Amlodipine/Perindopril (S05153) } & \\
\hline Individual Study Table Referring to Part of the Dossier & Volume: & Page: \\
\hline
\end{tabular}

Title of study: An Open-Label Study to Assess the Safety and Efficacy of Atorvastatin/Amlodipine/Perindopril Fixed-dose Combination in Adult Patients, Following Four Weeks of Adequate Treatment With Atorvastatin, Amlodipine and Perindopril Given Concurrently.

Protocol No.: CL3-05153-008

\section{Study centres:}

Five centres located in Vietnam enrolled a total of 130 patients.

\section{Publication (reference): Nil}

\section{Studied period:}

Initiation date: $19-A$ pr-2016

Completion date: 13 -Dec-2016

Phase of development of the study:

III/Local study for product registration in Vietnam

\section{Objectives:}

\section{Primary Objectives:}

Safety:

To assess the safety of Atorvastatin/Amlodipine/Perindopril fixed-dose combination (FDC) treatment.

\section{Efficacy:}

To assess the efficacy of the Atorvastatin/Amlodipine/Perindopril FDC treatment 10/5/5 mg and 20/5/5 mg strengths in maintaining blood pressure (BP) target (office based measurement) $<140 / 90 \mathrm{mmHg}$ (at Week 12) in patients previously treated with the free combination (FC) of atorvastatin, amlodipine and perindopril given concurrently at the same dose level as in the combination and controlled in the BP after 4 Weeks of treatment with the free combination.

\section{Secondary Objectives:}

Safety:

To assess the safety of atorvastatin, amlodipine and perindopril free combination.

\section{Efficacy:}

- To assess the efficacy of the concomitant administration of atorvastatin, amlodipine and perindopril free combination followed by FDC after 12-week treatment:

$\circ$ on reduction of Systolic Blood Pressure (SBP) and/or Diastolic Blood Pressure (DBP) (officebased measurement) versus Baseline.

o on percentage change and absolute change of low-density lipoprotein cholesterol (LDL-C) versus Baseline and on percentage of patients maintaining or achieving LDL-C target $(<100$ $\mathrm{mg} / \mathrm{dL})$.

a) To assess the efficacy of the Atorvastatin/Amlodipine/Perindopril FDC treatment 20/5/10 $\mathrm{mg}$ in achieving BP target (office based measurement) $<140 / 90 \mathrm{~mm} \mathrm{Hg}$ in patients previously treated with the free combination of atorvastatin 10 or $20 \mathrm{mg}$, amlodipine $5 \mathrm{mg}$ and perindopril $5 \mathrm{mg}$ given concurrently for 4 weeks and their BP not controlled (office based measurement: SBP $\geq 140 \mathrm{mmHg}$ or DBP $\geq 90$ $\mathrm{mmHg}$ ) at Week 4.

Methodology: 
This was a phase III, randomised, open-label, multicenter study for product registration in Vietnam, conducted in patients with hypertension and dyslipidemia. The duration of each patient in the study was planned for around 12 weeks. The eligible patients were included into the study at Visit 1 (Week 0) as per the inclusion criteria within 1 week from the Selection Visit date, and began a 4-week free combination period, followed by 8-week FDC period. This study was performed in strict accordance with Good Clinical Practice including the archiving of essential documents.

\section{Number of patients:}

Planned number of included patients: 132 patients were planned to be randomised

Actual number of included: 130 patients were randomised. Sixty seven patients were assigned to free combination $10+5+5$ group and 63 patients were assigned to free combination $20+5+5$ group.

\section{Diagnosis and main criteria for inclusion:}

\section{Selection criteria:}

The patients who met selection criteria were known hypertensive patients of 18 years of age or above, receiving monotherapy at initial dose for at least 1 month prior to Visit 0 and with inadequate BP control (office-based measurement): for either combined systolic and diastolic hypertension $(140 \mathrm{mmHg} \leq \mathrm{SBP}<160 \mathrm{mmHg}$ and 90 $\mathrm{mmHg} \leq \mathrm{DBP}<100 \mathrm{mmHg})$ or isolated systolic hypertension $(140 \mathrm{mmHg} \leq \mathrm{SBP}<160 \mathrm{mmHg})$ at Visit 0 .

Patients were selected if their LDL-C levels (according to a previous laboratory result within 3 months) were within the following ranges:

- $\quad$ LDL-C $<100 \mathrm{mg} / \mathrm{dL}(2.6 \mathrm{mmol} / \mathrm{L})$ if currently treated with atorvastatin $10 \mathrm{mg}$ or simvastatin 10 $\mathrm{mg}$ for less than 3 months, OR

- $\quad 100 \mathrm{mg} / \mathrm{dL}(2.6 \mathrm{mmol} / \mathrm{L}) \leq \mathrm{LDL}-\mathrm{C} \leq 190 \mathrm{mg} / \mathrm{dL}(4.9 \mathrm{mmol} / \mathrm{L})$ if patient naïve of statin treatment or currently treated with atorvastatin $10 \mathrm{mg}$ or simvastatin 5 or $10 \mathrm{mg}$ for less than 3 months.

\section{Inclusion criteria:}

Inclusion criteria were confirmed inadequate BP control before study drug dispensing (combined systolic and diastolic hypertension or isolated systolic hypertension) and LDL-C value of either $<100 \mathrm{mg} / \mathrm{dL}(2.6 \mathrm{mmol} / \mathrm{L})$ when currently treated with atorvastatin $10 \mathrm{mg}$ or simvastatin $10 \mathrm{mg}$ for less than 3 months or between 100 $\mathrm{mg} / \mathrm{dL}(2.6 \mathrm{mmol} / \mathrm{L})$ to $190 \mathrm{mg} / \mathrm{dL}(4.9 \mathrm{mmol} / \mathrm{L})$ both inclusive, in a statin naïve patient or patients currently treated with atorvastatin $10 \mathrm{mg}$ or simvastatin 5 or $10 \mathrm{mg}$ for less than 3 months.

\section{Non-selection criteria:}

The main non-selection criteria were known symptomatic orthostatic hypotension, malignant hypertension, secondary hypertension, isolated diastolic hypertension, hypertensive complications, diabetes mellitus type I or type II, history of malignancy in the last 5 years, any history of heart failure (New York Heart Association (NYHA) classification III or IV), clinically significant cardiac valvular disease, severe coronary heart disease as manifested by a history of myocardial infarction or unstable angina in the last 6 months prior to randomization, severe rhythm or conduction disorder and cerebrovascular ischemic event. Patients with history of haemorrhage within the previous 12 months, contra-indications to statins, to calcium channel inhibitors, to Angiotensin Converting Enzyme (ACE) inhibitors and history or current presence of lymphedema or leg oedema (unilateral or bilateral) of venous origin were precluded from study entry. Patients previously treated in the month before with perindopril $10 \mathrm{mg}$ or amlodipine $10 \mathrm{mg}$ and statin at the dose higher than lowest dose, patients with obesity, endocrine diseases, homozygous familial and heterozygous familial hypercholesterolemia, history of myopathy, alcohol or drug abuse and/or dependence, participation in another clinical study during the last 3 months prior to Visit 0 were also considered ineligible for study participation.

\section{Non-inclusion criteria:}

Non respect of non-selection criteria, results of laboratory examinations performed at the selection visit with clinically significant abnormalities that could affect the participation of the patient in the study, triglycerides $>400 \mathrm{mg} / \mathrm{dL}$, creatinine phosphokinase (CPK) levels $>5 \mathrm{x}$ upper limit of normal (ULN) or $>3 \times$ ULN with muscle pain, tenderness or weakness, uncorrected hypokalaemia or hyperkalaemia: potassium outside 3.0$5.0 \mathrm{mmol} / \mathrm{L}$; AST/ALT levels $>3 \mathrm{x} \mathrm{ULN}$; serum creatinine $>2 \times \mathrm{ULN}$, results of electrocardiogram (ECG) and echocardiography obtained from selection visit with clinically significant abnormalities that could affect the participation of the patient in the study were the main non-inclusion criteria. 


\section{Test drug:}

S 05153 (FDC of atorvastatin/amlodipine/perindopril): 10/5/5 milligram (mg) tablets, 20/5/5 mg and 20/5/10 mg tablets; oral administration, one tablet once daily (o.d.) before breakfast.

Batch No.:

$\mathrm{S} 05153$ 10/5/5 mg = L0058845, S 05153 20/5/5 mg = L0059332, S 05153 20/5/10 mg = L0060511

\section{Comparator (Reference product and/or placebo):}

Free combination of atorvastatin $10 \mathrm{mg}$, amlodipine $5 \mathrm{mg}$, and perindopril $5 \mathrm{mg}$ tablets or free combination of atorvastatin $20 \mathrm{mg}$, amlodipine $5 \mathrm{mg}$, and perindopril $5 \mathrm{mg}$ tablets. Oral administration, o.d. before breakfast. Batch No.:

Atorvastatin $10 \mathrm{mg}=$ L0060493, Atorvastatin $20 \mathrm{mg}=$ L0060491, Amlodipine $5 \mathrm{mg}=$ L0060467, Perindopril 5 $\mathrm{mg}=\mathrm{L} 0060386$

\section{Duration of treatment:}

\section{2-week treatment period:}

- $\quad$ The first 4-week period: at inclusion, patients were randomised to receive the free combination of atorvastatin, amlodipine and perindopril, either at the dose of $10 \mathrm{mg}, 5 \mathrm{mg}$ and $5 \mathrm{mg}$, respectively, or $20 \mathrm{mg}$, $5 \mathrm{mg}$ and $5 \mathrm{mg}$, respectively.

- $\quad$ The 8-weeks of fixed-dose combination period :

At Week 4, patients with adequate BP control (SBP $<140 \mathrm{mmHg}$ and DBP $<90 \mathrm{mmHg}$ ) were switched to the fixed-dose combination at the same dose levels as with the free combination: Atorvastatin/Amlodipine/Perindopril FDC 10/5/5 mg or Atorvastatin/Amlodipine/Perindopril FDC 20/5/5 mg. Any patient having $140 \mathrm{mmHg} \leq \mathrm{SBP}<160 \mathrm{mmHg}$ and/or $90 \mathrm{mmHg} \leq \mathrm{DBP}<100 \mathrm{mmHg}$ was up-titrated to Atorvastatin/Amlodipine/Perindopril FDC 20/5/10 mg

At Week 8, if BP was controlled (SBP $<140 \mathrm{mmHg}$ and $\mathrm{DBP}<90 \mathrm{mmHg}$ ), patients were continued on the same dosage. If BP was not controlled $(140 \mathrm{mmHg} \leq \mathrm{SBP} \leq 160 \mathrm{mmHg}$ and $90 \mathrm{mmHg} \leq \mathrm{DBP} \leq 100$ $\mathrm{mmHg}$ (combined hypertension) or $140 \mathrm{mmHg} \leq \mathrm{SBP} \leq 160 \mathrm{mmHg}$ or $\mathrm{DBP}<90 \mathrm{mmHg}$ (isolated systolic hypertension)), the dose was up-titrated to FDC of Atorvastatin/Amlodipine/Perindopril 20/5/10 mg.

If office-based SBP $>160 \mathrm{mmHg}$ or DBP $>100 \mathrm{mmHg}$ at the end of Week 4 (Visit 2) or at the end of Week 8 (Visit 3), patient should be withdrawn.

\section{Criteria for evaluation:}

The primary safety endpoint was the number and percentage of patients reporting at least one adverse event (AE) during the usage of either FDC $10 / 5 / 5$ or 20/5/5; or 20/5/10 mg. AEs were assessed from inclusion till end of the study i.e. at Weeks $0,4,8$ and 12 visits. To analyze AEs during usage of FDC, AEs from Week 4 up to end of study were considered.

The primary efficacy endpoint was the percentage of patients who, after 8 weeks of the FDC treatment with $10 / 5 / 5$ or $20 / 5 / 5 \mathrm{mg}$ strengths, maintained the BP (office based measurements) $<140 / 90 \mathrm{mmHg}$ and were previously treated with the free combination of atorvastatin, amlodipine and perindopril given concurrently at the same dose level as in the combination and controlled on the BP after 4 weeks of treatment with the free combination.

The secondary endpoints were:

\section{Safety measurements:}

- $\quad$ The number and percentage of patients reporting at least one AE during the usage of either $\mathrm{FC} 10+5+5$ or $20+5+5 \mathrm{mg}$.

- Other safety measurements: vital signs, clinical laboratory parameters (biochemistry and haematology), electrocardiogram

\section{Efficacy measurements:}

i. The change in SBP and DBP (office based measurement) from baseline (Week 0) to Week 12 for patients having same dosage of atorvastatin, amlodipine and perindopril

ii. The percentage change in LDL-C from baseline (Week 0) to Week 12 for patients having same dosage 
of atorvastatin, amlodipine and perindopril (descriptive analysis for Group 1 (Patients having free combination of atorvastatin/amlodipine/perindopril $10+5+5 \mathrm{mg}$ in 4 weeks, then fixed dose combination of Atorvastatin/Amlodipine/Perindopril 10/5/5 $\mathrm{mg}$ in 8 weeks) and Group 2 (Patients having free combination of atorvastatin/amlodipine/perindopril $20+5+5 \mathrm{mg}$ in 4 weeks, then fixed dose combination of Atorvastatin/Amlodipine/Perindopril 20/5/5 mg in 8 weeks) separately).

iii. Absolute change in LDL-C from baseline (Week 0) to Week 12 for patients having same dosage of atorvastatin, amlodipine and perindopril

iv. Percentage of patients maintaining or achieving LDL-C target $(<100 \mathrm{mg} / \mathrm{dL})$

v. The percentage of patients who, after 4 or 8 weeks of FDC treatment with 20/5/10 mg strength, achieved the BP (office-based measurement) $<140 / 90 \mathrm{mmHg}$ in patients previously uncontrolled with FC/FDC of atorvastatin, amlodipine and perindopril 10/5/5 $\mathrm{mg}$ or $20 / 5 / 5 \mathrm{mg}$

\section{Statistical methods: \\ Analysis Sets: \\ Safety Analysis Set:}

The patients who received at least one dose of study medication were included in the safety analysis. The safety population comprised of: safety set for free combination $\left(\mathrm{SS}_{\mathrm{FC}}\right)$, with at least one dose of free combination during Week 0 to Week 4 (either $10+5+5$ or $20+5+5 \mathrm{mg}$ ) and safety set for fixed dose combination $\left(\mathrm{SS}_{\mathrm{FDC}}\right)$, with at least one dose of FDC during Week 4 to Week 12 (either 10/5/5; 20/5/5 or 20/5/10 mg).

Randomised Set:

Randomised set (RS) was defined as all patients to whom a therapeutic unit was assigned at Week 0 visit (V1) during randomization process.

Enrolled population:

Enrolled population included patients who signed informed consent form.

Intention-to-Treat (ITT) Analysis Set:

The main analysis set, defined as all patients who received at least one dose of the study medication and had at least an analyzable value at Week 4 and one post Week 4 of mean sitting SBP and DBP, was the ITT population. This population was used for all efficacy evaluations and was considered as primary population.

Per Protocol (PP) Analysis Set:

The secondary analysis set, the Per Protocol (PP) set, excluded all patients of the ITT population with relevant deviation which affected evaluation of the primary efficacy endpoint.

\section{Efficacy analysis:}

Primary efficacy analysis:

Frequency and percentage of patients who, after 8 weeks of FDC treatment with 10/5/5 or 20/5/5 mg strengths, maintained the BP (office-based measurement) $<140 / 90 \mathrm{mmHg}$ was calculated.

Secondary efficacy analyses:

- Change in SBP and DBP and percentage and absolute change in LDL-C from baseline (Week 0 ) to Week 12 after receiving either $10 / 5 / 5 \mathrm{mg}$ or $20 / 5 / 5 \mathrm{mg}$ in both free combination and FDC period were summarized descriptively.

- Percentage of patients who, after 4 or 8 weeks of FDC treatment with 20/5/10 mg strength, achieved the BP (office-based measurement) $<140 / 90 \mathrm{mmHg}$ in patients previously uncontrolled with the free combination or FDC of Atorvastatin 10 or $20 \mathrm{mg}$, Amlodipine $5 \mathrm{mg}$ and Perindopril $5 \mathrm{mg}$ were summarized as frequency and percentage.

Other efficacy analyses:

i. Change in the SBP and DBP from baseline (Week 0) to each Visit for patients receiving either 10/5/5 mg or 
20/5/5 mg in both free combination and FDC period was summarized descriptively.

ii. Percentage and absolute change in lipid parameters- from baseline (Week 0) to Week 12 for patients receiving either $10 / 5 / 5 \mathrm{mg}$ or $20 / 5 / 5 \mathrm{mg}$ in both free combination and FDC period were summarized descriptively.

Study outcome and safety analysis:

Safety was monitored by assessing the incidence of AEs, vital signs, physical examination and laboratory test values at each visit. The incidence of symptomatic hypotension, angioedema, myalgia and elevated liver enzymes were particularly focused. Number and percentage of treatment emergent AEs and SAEs were tabulated with respect to the intensity, outcome, relationship to the study products, and AEs leading to the study drug withdrawal. The SAEs were listed and discussed separately.

Individual data listings of laboratory results and vital signs were presented for each patient at all visits (scheduled and non-scheduled). The results of 12-lead or 3-lead ECG were summarized by protocol specified time points and study medication dosage exposure.

\section{SUMMARY - CONCLUSIONS}

\section{DISPOSITION OF PATIENTS AND ANALYSIS SETS}

\begin{tabular}{|c|c|c|c|}
\hline Patient Disposition & $\begin{array}{l}\mathrm{FC}: 10+5+5 \\
(\mathrm{~N}=67)\end{array}$ & $\begin{array}{l}\mathrm{FC}: 20+5+5 \\
(\mathrm{~N}=63)\end{array}$ & $\begin{array}{l}\text { Total } \\
(\mathrm{N}=130)\end{array}$ \\
\hline Number of Patients Randomized & $67(100.0 \%)$ & $63(100.0 \%)$ & $130(100.0 \%)$ \\
\hline Number of Patients Treated & $67(100.0 \%)$ & $63(100.0 \%)$ & $130(100.0 \%)$ \\
\hline $\begin{array}{l}\text { Number of Patients who Completed } \\
\text { the Study }\end{array}$ & $54(80.6 \%)$ & $53(84.1 \%)$ & $107(82.3 \%)$ \\
\hline $\begin{array}{l}\text { Number of Patients Treated but } \\
\text { Discontinued before Study Completion } \\
\text { (Early withdrawal) }\end{array}$ & $13(19.4 \%)$ & $10(15.9 \%)$ & $23(17.7 \%)$ \\
\hline \multicolumn{4}{|l|}{ Reason for Early Withdrawal } \\
\hline Adverse Events related & $6(9.0 \%)$ & $3(4.8 \%)$ & $9(6.9 \%)$ \\
\hline Lack of efficacy & $0(0.0 \%)$ & $2(3.2 \%)$ & $2(1.5 \%)$ \\
\hline Patient consent withdrawal & $7(10.4 \%)$ & $5(7.9 \%)$ & $12(9.2 \%)$ \\
\hline Safety Population & $67(100.0 \%)$ & $63(100.0 \%)$ & $130(100.0 \%)$ \\
\hline \begin{tabular}{|cccc} 
Safety Analysis Set for & Free \\
Combination: SS & & & \\
Co
\end{tabular} & $67(100.0 \%)$ & $63(100.0 \%)$ & $130(100.0 \%)$ \\
\hline $\begin{array}{c}\text { Safety Analysis Set for Free } \\
\text { Combination: SSFDC }\end{array}$ & $60(89.6 \%)$ & $57(90.5 \%)$ & $117(90.0 \%)$ \\
\hline ITT Population & $58(86.6 \%)$ & $57(90.5 \%)$ & $115(88.5 \%)$ \\
\hline PP Population & $47(70.1 \%)$ & $51(81.0 \%)$ & $98(75.4 \%)$ \\
\hline
\end{tabular}

\section{BASELINE CHARACTERISTICS}

Majority of patients in this study belonged to the age group of $\geq 18$ to $<65$ years with similar gender distribution across all groups. All patients were Asians, hypertensive with inadequate BP control and were on prior antihypertensive monotherapy for at least one month as per the inclusion criteria. These patients also had elevated LDL-C or were on statin therapy. Essential hypertension was present for approximately 4 years while dyslipidemia was of current onset and less than 4 months duration. Around $50 \%$ of study population presented with hypercholesterolemia which was more common in FC $10+5+5 \mathrm{mg}$ group $(62.7 \%)$ as compared to $\mathrm{FC}$ $20+5+5 \mathrm{mg}$ group $(41.3 \%)$. More than two thirds of the study population was non-smoker and had no habit of alcohol drinking. The mean SBP, DBP and LDL-C were comparable across FC 10+5+5 mg and FC $20+5+5 \mathrm{mg}$ group in the randomised set. Overall, the patients confirmed to the target population.

Out of 152 patients screened, 130 patients were randomised in the study: 67 patients were assigned to FC 
10+5+5 $\mathrm{mg}$ group and 63 patients were assigned to FC 20+5+5 mg group. Amongst 117 patients who received FDC, 115 formed ITT dataset and 98 constituted PP dataset. The number of patients that needed to be uptitrated was less than one-third of the total population as assumed. A total of 107 patients completed the study.

\section{EXTENT OF EXPOSURE}

Overall, the mean drug compliance was more than $96 \%$ in all treatment groups. Additionally, $>85 \%$ of patients in all groups showed treatment compliance between $80-120 \%$.

The drug exposure correlated with the expected treatment duration of the respective groups; mean drug exposure during treatment with free combinations was around 30 days, during treatment with fixed dose combination was around 56 days and during entire study duration was around 80 days.

\section{EFFICACY RESULTS}

\section{- Primary assessment criterion}

Thirty eight (86.4\%) of 44 patients in Group 1 and 40 (90.9\%) of 44 patients in Group 2 from the ITT population maintained the BP below 140/90 mmHg at Week 12. Patients who did not maintain the BP target (except those who had missing values) were close to the target value of $<140 / 90 \mathrm{mmHg}$. These observations were also noted in the PP dataset.

- Secondary assessment criteria

In the ITT population, the mean (SD) reduction in SBP was $-27.34( \pm 11.6) \mathrm{mmHg}$ in Group 1 and $-24.45( \pm 9.4)$ $\mathrm{mmHg}$ in Group 2. In the PP population, the mean (SD) reductions in SBP in Group 1 and Group 2 were -27.71 $( \pm 12.4) \mathrm{mmHg}$ and $-24.52( \pm 9.5) \mathrm{mmHg}$, respectively. In the ITT population, the mean (SD) reductions in the DBP were $-12.39( \pm 9.5) \mathrm{mmHg}$ and $-9.95( \pm 8.9) \mathrm{mmHg}$ in Group 1 and Group 2, respectively. In the PP population the mean (SD) reductions in DBP in Group 1 and Group 2 were $-11.68( \pm 10.0) \mathrm{mmHg}$ and -10.01 $( \pm 9.0) \mathrm{mmHg}$, respectively.

There was a mean $(\mathrm{SD})$ percentage reduction of $-28.36( \pm 22.9) \%$ and $-32.76( \pm 32.7) \%$ in LDL-C from baseline to Week 12 in the groups 1 and 2 from the ITT population, respectively. Group 1 had a mean (SD) reduction in the absolute value of LDL-C by $-39.33( \pm 31.1) \mathrm{mg} / \mathrm{dl}$ and Group 2 had a mean (SD) reduction in the absolute value of LDL-C by $-45.8( \pm 37.1) \mathrm{mg} / \mathrm{dl}$ from baseline to Week 12 . Similar changes in the percentage and absolute values of LDL-C were observed in the PP population. The difference observed was expected as the patients in Group 1 received atorvastatin $10 \mathrm{mg}$ and patients in Group 2 received atorvastatin $20 \mathrm{mg}$.

More than 85\% patients from Group 2 and 4, who received Atorvastatin $20 \mathrm{mg}$ through the entire study period achieved LDL-C target $(<100 \mathrm{mg} / \mathrm{dL})$. There was reduction of at least $-27.47 \%$ in LDL-C across all the groups.

All patients in Groups 3 and 4 who received FDC 20/5/10 mg after uptitration at W4 and in Group 6 who received FDC 20/5/10 mg after uptitration at W8 achieved BP target of $<140 / 90 \mathrm{mmHg}$ after 4 weeks of treatment with the fixed dose combination. Two out of 3 patients in Group 5 who received FDC 20/5/10 mg after uptitration at W8 achieved BP target of $<140 / 90 \mathrm{mmHg}$ after 4 weeks of treatment with the fixed dose combination.

There was a reduction in the total cholesterol in all the groups from baseline to Week 12. Increase in HDL-C was observed in all groups except in Groups 1 and 5. There was a variable reduction in the triglyceride level from baseline to Week 12, with a greater amplitude in the group using atorvastatin $20 \mathrm{mg}$.

\section{SAFETY RESULTS}

\section{- Emergent adverse events}

Overall, a total of 32 Treatment Emergent Adverse Events (TEAEs) and 2 ERIN of overdose were reported in 27 $(20.8 \%)$ patients. The frequency and percentage of TEAEs were comparable between $\mathrm{SS}_{\mathrm{FC}} 10+5+5 \mathrm{mg}$ and $\mathrm{SS}_{\mathrm{FC}}$ 20+5+5 mg: 7 TEAEs and 2 ERIN in 8 patients (11.9\%) versus 8 TEAEs in seven patients $(11.1 \%)$, respectively. In $\mathrm{SS}_{\mathrm{FDC}}$, there were 17 TEAEs reported across all groups: 4 in $\mathrm{SS}_{\mathrm{FDC}}$ 10/5/5 mg (W4-W12), 7 in SS $\mathrm{SDC}_{\text {20/5/5 }}$ $\mathrm{mg}$ (W4-W12), 4 in FDC 20/5/10 mg (W4-W12) and 2 in SS FDC 10/5/5 mg then 20/5/10 mg (W4-W12). None was reported in $\mathrm{SS}_{\mathrm{FDC}} 20 / 5 / 5 \mathrm{mg}$ then $20 / 5 / 10 \mathrm{mg}$ (W4-W12) group. The most frequently reported TEAEs ( $\geq 2$ events) were cough ( 3 events), pharyngitis ( 2 events) and ALT increased ( 2 events). All the TEAEs noted were of grade 1 (mild) or grade 2 (moderate) intensity. Of these 32 TEAEs and 2 ERIN, 19 were assessed to be related to study medication: 9 TEAEs reported in the $\mathrm{SS}_{\mathrm{FC}}$ and 10 TEAEs in the SS $\mathrm{FDC}_{\text {groups. Eleven TEAEs resulted }}$ in the discontinuation of the patients from the study with 7 TEAEs reported in the $\mathrm{SS}_{\mathrm{FC}}$ and 4 TEAEs in the 
SS

\section{- Laboratory tests}

Two events of increased ALT at Weeks 4 and 8, which were considered as related events led to discontinuation of the patients from the study. None of the other laboratory changes resulted in early termination of the patient from the study.

\section{- Other safety evaluation}

No clinically significant changes were observed in the physical examination, vital signs and ECG.

\section{CONCLUSION}

Overall, the fixed drug combination of Atorvastatin/Amlodipine/Perindopril was effective in maintaining the BP target $<140 / 90 \mathrm{mmHg}$. There was a reduction in mean SBP and DBP, by approximately 25 and $10 \mathrm{mmHg}$, respectively after they were treated with the FDC 10/5/5 and 20/5/5 mg. Additionally, higher dose strength of $20 / 5 / 10 \mathrm{mg}$ was found to be useful in achieving BP target in around $2 \backslash 3^{\text {rd }}$ of patients who required uptitration. FDC of Atorvastatin $\backslash$ Amlodipine $\backslash$ Perindopril led to reduction in LDL-C in all dose strengths, with a greater extent in the patients who received atorvastatin $20 \mathrm{mg}$ (up to $40 \mathrm{mg} / \mathrm{dL}$ ). Favourable changes in the total cholesterol, HDL-C and triglycerides were also noted coupled with better adherence to the fixed drug combination. Safety profile of free combination $\mathrm{SS}_{\mathrm{FC}} 10+5+5 \mathrm{mg}$ was similar to that of free combination $\mathrm{SS}_{\mathrm{FC}}$ $20+5+5 \mathrm{mg}$ and safety profiles of the fixed dose combinations in this study were comparable between groups. Fixed dose combination of Atorvastatin/Amlodipine/Perindopril at the doses of 10/5/5 mg, 20/5/5 $\mathrm{mg}$ and $20 / 5 / 10 \mathrm{mg}$ were well tolerated by the study population and found to be safe for administration in the Vietnamese patients.

Date of the report: $24-\mathrm{Aug}-2017$

Version of the report: V 1.0 